\title{
Field and in vitro insecticidal efficacy of alphacypermethrin-treated high density polyethylene mesh against Culicoides biting midges in South Africa
}

\author{
P.C. Page ${ }^{a, *}$, K. Labuschagne ${ }^{\text {b }}$, G.J. Venter ${ }^{\text {b }}$, J.P. Schoeman ${ }^{\text {a }}$, A.J. Guthrie ${ }^{c}$ \\ a Department of Companion Animal Clinical Studies, Faculty of Veterinary Science, University of Pretoria, \\ Private Bag X04, Onderstepoort 0110, South Africa \\ ${ }^{\mathrm{b}}$ PVVD, ARC-Onderstepoort Veterinary Institute, Private Bag X05, Onderstepoort 0110, South Africa \\ c Equine Research Centre, Faculty of Veterinary Science, University of Pretoria, Private Bag X04, \\ Onderstepoort 0110, South Africa
}

\section{ABSTRACT}

The efficacy of untreated and alphacypermethrin-treated high density polyethylene (HDPE) mesh against Culicoides biting midges (Diptera: Ceratopogonidae) was determined using Onderstepoort downdraught black light traps and a contact bioassay. Three traps were operated overnight in four replicates of a 3 x 3 randomised Latin square design near horses under South African field conditions. Both the untreated and alphacypermethrin-treated HDPE mesh significantly $(P<0.05)$ reduced the numbers of Culicoides midges, predominantly Culicoides (Avaritia) imicola Kieffer, collected in the light traps by 4.2 and 7.2 times respectively. A repellent effect of the alphacypermethrin-treated mesh was not confirmed because the number of midges collected in the light traps with untreated and alphacypermethrin-treated HDPE mesh were not significantly different $(P=0.656)$. Bioassay of the insecticidal contact efficacy indicated median C. imicola mortality of $100 \%$ from 30 and 10 min following exposure to the alphacypermethrin-treated HDPE mesh for 1 or $3 \mathrm{~min}$, respectively. In the bioassay, mortality was significantly higher $(P=0.016)$ at $5 \mathrm{~min}$ post exposure in the midges exposed to the alphacypermethrin-treated mesh for $3 \mathrm{~min}(74.8 \%)$ compared to the $1 \mathrm{~min}$ exposure group (59.5\%). The HDPE mesh could be used to reduce exposure of housed animals to Culicoides midges, specifically $C$. imicola, and viruses transmitted by these midges. Mesh treated with alphacypermethrin had the additional benefit of a rapid insecticidal effect on $C$. imicola. 
Keywords: C. imicola, contact bioassay, light trap, synthetic pyrethroid, African horse sickness

\section{Introduction}

Culicoides biting midges (Diptera: Ceratopogonidae) are of economic and veterinary significance worldwide, primarily due to the Orbiviruses they transmit (Mellor et al., 2000; Meiswinkel et al., 2004). Based on its wide geographical distribution and host preference for bigger mammals, as indicated by the high numbers collected near livestock, Culicoides (Avaritia) imicola Kieffer is considered the principal vector of African horse sickness virus (AHSV), equine encephalosis virus and bluetongue virus (BTV) in South Africa (Nevill et al., 1992; Meiswinkel et al., 2004; Paweska and Venter, 2004), and BTV in southern Europe (Mellor, 1992; Mellor et al., 2000). Culicoides imicola is predominant on livestock throughout South Africa and is also the most abundant species collected on horses at Onderstepoort, South Africa (Scheffer et al., 2012).

The spread of BTV in northern Europe and recent outbreaks of a novel orthobunyavirus, Schmallenberg virus (Hoffmann et al., 2012), has demonstrated the devastating effect of these viruses on naïve populations. These outbreaks raised concern over the introduction and spread of other midge-borne viruses, particularly AHSV, and the need for optimised preventative strategies (Carpenter et al., 2008; Carpenter et al., 2009; MacLachlan and Guthrie, 2010; Papadopoulos et al., 2010; Backer and Nodelijk, 2011; Tarlinton et al., 2012; MacLachlan and Mayo, 2013; Napp et al., 2013). Moreover, global expansion of containerised trade, including intercontinental movement of horses (Reiter, 2010), provides potential mechanisms whereby viruses may be introduced (Carpenter et al., 2009; MacLachlan and Guthrie, 2010; de Vos et al., 2012; Napp et al., 2013). Whilst clear recommendations for pre-export quarantine and testing of horses for AHSV have been in place for many years, recent amendments to the World Organisation for Animal Health (OIE) - Terrestrial Animal Health Code have included recommendations that mesh of appropriate gauge, impregnated 
with an approved insecticide be placed over containers during transport of horses through regions not free of AHSV (World Organisation for Animal Health, 2013).

In addition to the use of vaccines, recommended control measures against viruses transmitted by Culicoides midges include stabling at night, screening of stables with mesh, and the use of effective repellents or insecticides (Meiswinkel et al., 2004; Carpenter et al., 2008). Stabling efficacy depends on the exophilic/endophilic behaviour of the vector species (Carpenter et al., 2008; Viennet et al., 2012), and horses are protected only if the stables are adequately closed (Barnard, 1997; Meiswinkel et al., 2000). Repellents such as N,N-diethyl-3-methylbenzamide (Page et al., 2009), pyrethroids and plant extracts (Braverman and Chizov-Ginzburg, 1998), and organic fatty acids (Venter et al., 2011) have shown efficacy against Culicoides midges in light trap studies. Recently, insecticidal efficacy against colony-reared Culicoides (Monoculicoides) nubeculosus Meigen exposed to hair from horses treated with cypermethrin was reported (Papadopoulos et al., 2010). Despite the proven effectiveness (Lengeler, 2004) and widespread use of pyrethroid-treated nets for controlling mosquitoes, the use of pyrethroid-treated mesh for reducing the Culicoides biting rate in animals has received limited attention (Carpenter et al., 2008; Calvete et al., 2010; Del Río et al., 2014).

The objectives of this study were to determine if alphacypermethrin-treated high density polyethylene (HDPE) mesh applied to light traps will reduce the entry of Culicoides midges, particularly $C$. imicola, into the traps. Following on the promising results of Papadopoulos et al. (2010) with cypermethrin and the northern European $C$. nubeculosus, the in vitro insecticidal efficacy of alphacypermethrin-treated HDPE mesh against field populations of C. imicola was investigated. These results may support potential of alphacypermethrin-treated HDPE nets for protecting livestock against Culicoides midges, and be applicable to containerised transport systems of horses. 


\section{Methods}

\subsection{Light trap assay}

The efficacy in reducing numbers of Culicoides midges entering a light trap of (1) a black, 400 denier, knitted monofilament HDPE mesh with $0.3 \mathrm{~mm}$ hole size (RK02 70\% Shade Cloth, Alnet, South Africa) treated with alphacypermethrin (Fendona ${ }^{\circledR} 6$, BASF Agro BV Arnhem, Switzerland), (2) an identical untreated HDPE mesh and (3) an untreated control black polyester mesh with $2 \mathrm{~mm}$ hole size were compared. Comparisons were done overnight for 12 nights in four replicates of a 3 X 3 randomized Latin square design (Snedecor and Cochran, 1980), observer-blinded, field experiment near horses at the Faculty of Veterinary Science, Onderstepoort $\left(25^{\circ} 38^{\prime} 51.42^{\prime \prime S}, 28^{\circ} 10^{\prime} 45.96^{\prime \prime} \mathrm{E}, 1\right.$ $238 \mathrm{~m}$ above sea level) during late summer between 27 March and 21 April 2011. The meshes replaced the standard white polyester netting around the light source of three $220 \mathrm{~V}$ down-draught Onderstepoort suction light traps equipped with $8 \mathrm{~W}, 23 \mathrm{~cm}$ black light tubes (Venter et al., 2009).

The alphacypermethrin-treated HDPE mesh was prepared according to the insecticide manufacturer's instructions for the treatment of bed nets against mosquitoes for a target dose of $20-40 \mathrm{mg} / \mathrm{m}^{2}$. The mesh was immersed in alphacypermethrin suspension for $30 \mathrm{~min}$, air dried overnight at $20^{\circ} \mathrm{C}$ and $65 \%$ relative humidity $(\mathrm{RH})$, then kept wrapped in tin foil prior to being attached to the light trap with elastic bands. A new mesh was prepared each day for each of the traps. Meshes were removed from the traps $14 \mathrm{~h}$ after application. Alphacypermethrin uptake was quantified by high performance liquid chromatography (HPLC) analysis of a duplicate mesh sample prepared for each replicate. The traps were placed in a row $6.5 \mathrm{~m}$ apart to minimise interference between traps (Venter et al., 2012), $2.0 \mathrm{~m}$ above the ground, and operated from before sunset (18h00) to after sunrise (06h00). Catches were made into $500 \mathrm{ml}$ plastic beakers containing $200 \mathrm{ml}$ 0.5\% Savlon ${ }^{\circledR}$ (Johnson and Johnson, South Africa) and water solution. Overnight insect collections were stored in $70 \%$ ethanol prior to the total number of Culicoides specimens and the number of $C$. imicola being determined. Climatic variables (outside temperature, RH, wind speed, rainfall) were recorded hourly using a weather station (Vantage Pro2, Davis, USA) and data logger (Weatherlink, Davis, USA). 


\subsection{Contact bioassay}

The in vitro insecticidal efficacy of meshes against field-collected, nulliparous (unpigmented) female (Dyce, 1969) C. imicola was assessed in a contact bioassay conducted between 23 and 27 April 2012. The midges had been collected the previous night in Onderstepoort light traps at the ARCOnderstepoort Veterinary Institute (253' S, 28 ${ }^{\circ} 11^{\prime} \mathrm{E} ; 1219 \mathrm{~m}$ above sea level) and were handled prior to the assay as previously described (Venter et al., 1998).

In the absence of laboratory colonies to standardise the physiological age of the midges only nulliparous $C$. imicola were used in the assays. Midges were immobilised for 1 min at $-4^{\circ} \mathrm{C}$ and groups of 10 nulliparous females aspirated into glass Pasteur pipettes on a refrigerated chill table. At the start of each exposure experiment, the 10 nulliparous females were expelled into one of $60 \times 12$ $\mathrm{mm}$ glass petri dishes (Anumbra, Lasec, South Africa) and exposed to a $19.6 \mathrm{~cm}^{2}$ alphacypermethrintreated HDPE mesh (prepared as described for the light trap assay) for 1 or $3 \mathrm{~min}$, or an untreated control HDPE mesh for 3 min. The meshes were attached to the petri dish by a small disc of adhesive putty (Prestik ${ }^{\circledR}$, Bostik, South Africa). To maximise contact with the mesh the dishes were gently inverted every $30 \mathrm{sec}$. Following the exposure period midges were immobilised for $1 \mathrm{~min}$ at $-4^{\circ} \mathrm{C}$ and the petri dish cover with the attached mesh was replaced with a clean cover to allow the midges to recover without residual exposure to insecticide. Mortalities were visually assessed at 5, 10, 30, 60 min and $24 \mathrm{~h}$ post exposure to the mesh by an entomologist blinded to the treatment group. Midges that were motionless or twitching and incapable of oriented movement were classified as dead. Following the $60 \mathrm{~min}$ assessment midges were maintained overnight at $24.5^{\circ} \mathrm{C}$ and $60 \% \mathrm{RH}$ until the final efficacy assessment at $24 \mathrm{~h}$. Thirty replicates of each exposure period were conducted.

\subsection{Statistical analyses}

Statistical analyses were done using SPSS ${ }^{\circledR}$ Statistics version 21 (IBM, USA). For the light trap assay midge numbers were natural logarithm-transformed to achieve normality. Mean numbers of Culicoides midges and C. imicola were compared between treatment groups while controlling for the effects of light trap location and day using analysis of variance (ANOVA). Where $F$-ratios were 
significant, pairwise comparisons were done between treatment groups using Tukey's HSD test. Homogeneity of variances was tested with Levene's test. Statistical testing was conducted at the 5\% level of significance.

Contact bioassay midge mortality, corrected for mortality in the untreated control group with Abbott's formula [Mortality $\%=100 \mathrm{x}(\mathrm{X}-\mathrm{Y}) / \mathrm{Y}$; where $\mathrm{X}=\%$ survival in the untreated control, $\mathrm{Y}=\%$ survival in the treated sample] (Abbott, 1925), was compared between groups at each time point by Kruskal-Wallis one-way ANOVA on ranks. The Mann-Whitney U test was used for pairwise comparisons. Post hoc comparisons were adjusted using the Bonferroni correction of $P$ values. When the Bonferroni correction was applied, $P<0.017$ was considered significant.

\section{Results}

\subsection{Light trap assay}

A total of 37221 Culicoides midges were collected in 36 collections made over 12 nights from three light traps operated simultaneously. Culicoides imicola was the most abundant species and comprised $96.1 \%$ of midges collected. The mean number of both Culicoides midges and $C$. imicola collected with the control trap were significantly higher than the alphacypermethrin-treated HDPE mesh $(P=$ 0.001 for both) and the untreated HDPE mesh light trap $(P<0.05$ for both) (Table 1). The smaller total number of Culicoides or $C$. imicola collected with the alphacypermethrin-treated HDPE mesh was not significantly different $(P=0.656)$ from that of the untreated HDPE mesh. The proportion of C. imicola in relation to the other species collected ranged from $97.1 \%$ with the untreated HDPE mesh to $95.9 \%$ in the control trap and was not significantly different between treatments $\left(X^{2}=1.717\right.$, d.f. $=$ 2, $P=0.424)($ Table 1). 
Table 1. Mean \pm s.e. number of Culicoides midges and $C$. imicola collected by three black light traps fitted with untreated HDPE mesh, alphacypermethrin-treated $\left(36.3 \pm 6.1 \mathrm{mg} / \mathrm{m}^{2}\right)$ HDPE mesh, or untreated control polyester mesh, operated overnight for 12 nights between 27 March and 21 April 2011.

\begin{tabular}{|c|c|c|c|}
\hline & \multicolumn{3}{|c|}{ Mesh treatment } \\
\hline & Untreated HDPE mesh & $\begin{array}{l}\text { Alphacypermethrin- } \\
\text { treated HDPE mesh }\end{array}$ & $\begin{array}{c}\text { Untreated control pol- } \\
\text { yester mesh }\end{array}$ \\
\hline Culicoides midges & $539 \pm 218^{a}$ & $314 \pm 102^{\mathrm{a}}$ & $2249 \pm 763^{b}$ \\
\hline C. imicola & $523 \pm 214^{\mathrm{a}}$ & $303 \pm 99^{\mathrm{a}}$ & $2156 \pm 743^{b}$ \\
\hline$\%$ C. imicola & 97.1 & 96.3 & 95.9 \\
\hline
\end{tabular}

Values within each row with a different superscript differ significantly $(P<0.05)$.

The mean \pm s.d. alphacypermethrin uptake by the treated HDPE meshes as determined by HPLC analysis was $36.3 \pm 12.3 \mathrm{mg} / \mathrm{m}^{2}$, within the target range of $20-40 \mathrm{mg} / \mathrm{m}^{2}$. The mean \pm s.d. outside temperature, $\mathrm{RH}$, wind speed, and rain during the light trap collection were $18.9 \pm 2.2^{\circ} \mathrm{C}, 79.7 \pm$ $7.2 \%, 1 \pm 0.4 \mathrm{~km} / \mathrm{h}$, and $7.2 \pm 16.4 \mathrm{~mm}$, respectively.

\subsection{Contact bioassay}

The median percentage efficacy of the alphacypermethrin-treated HDPE mesh in both the 1 min and 3 min exposure groups was significantly $(P<0.001)$ higher than the untreated control HDPE mesh at all the time points tested (Table 2). Median C. imicola mortality was $100 \%$ from 30 and 10 min following exposure to the alphacypermethrin-treated HDPE mesh of 1 and 3 min, respectively. The median mortality was significantly higher $(P=0.016)$ in the 3 min alphacypermethrin exposure group (74.8\%) compared to the $1 \mathrm{~min}$ exposure group $(59.5 \%)$ at the $5 \mathrm{~min}$ time point only. 
Table 2. Median (IQR) percentage mortality of field-collected nulliparous (unpigmented), female Culicoides imicola exposed to alphacypermethrin-treated HDPE mesh for 1 or $3 \mathrm{~min}$, or to an untreated control HDPE mesh for $3 \mathrm{~min}$ in a contact bioassay. Values for the alphacypermethrin-treated HDPE mesh exposure have been corrected with Abbott's formula. Thirty replicates of each exposure period were conducted between 23 and 27 April 2012.

\begin{tabular}{|c|c|c|c|}
\hline \multirow{3}{*}{ Time post exposure } & \multicolumn{3}{|c|}{ Exposure } \\
\hline & \multicolumn{2}{|c|}{ Alphacypermethrin-treated HDPE mesh } & \multirow{2}{*}{$\begin{array}{c}\text { Untreated control } \\
3 \mathrm{~min}\end{array}$} \\
\hline & $1 \mathrm{~min}$ & $3 \mathrm{~min}$ & \\
\hline $5 \min$ & $59.5^{a^{*}}(35.6-80.3)$ & $74.8^{b^{*}}(62.8-100)$ & $11.1^{\mathrm{c}}(0.0-29.8)$ \\
\hline $10 \mathrm{~min}$ & $84.7^{\mathrm{a}}(72.0-100)$ & $100^{\mathrm{a}}(82.5-100)$ & $12.5^{\mathrm{b}}(10.0-29.8)$ \\
\hline $30 \mathrm{~min}$ & $100^{\mathrm{a}}(100-100)$ & $100^{\mathrm{a}}(100-100)$ & $11.1^{\mathrm{b}}(0.0-38.1)$ \\
\hline $60 \mathrm{~min}$ & $100^{\mathrm{a}}(100-100)$ & $100^{\mathrm{a}}(100-100)$ & $20.0^{\mathrm{b}}(10.0-29.8)$ \\
\hline $24 \mathrm{~h}$ & $100^{\mathrm{a}}(100-100)$ & $100^{\mathrm{a}}(100-100)$ & $57.1^{\mathrm{b}}(36.5-77.8)$ \\
\hline
\end{tabular}

Values within each row with a different superscript differ significantly $(P<0.001)$. * indicates $P=0.016$.

\section{Discussion}

The HDPE mesh had a significant effect in reducing the numbers of Culicoides midges, predominantly $C$. imicola, collected by the light trap. The magnitude of reduction for the untreated HDPE mesh and alphacypermethrin-treated HDPE mesh was 4.2 and 7.2 times, respectively, with efficacy likely related to the smaller hole size of the HDPE mesh, compared to the untreated control polyester mesh. Whilst the light trap with the alphacypermethrin-treated mesh consistently collected fewer Culicoides midges than the untreated HDPE mesh, a significant repellent effect was not demonstrated. This is in agreement with a previous study at Onderstepoort where no repellent effect 
against Culicoides midges was demonstrated for a different alphacypermethrin formulation tested at a lower concentration $(0.3 \%)$ (Page et al., 2009). However, a limitation of using light traps alone to screen mesh for repellent and insecticidal efficacy against Culicoides midges is that the relative strong attractant effect of the light may override any repellent effect (Page et al., 2009; Venter et al., 2009) and a large mesh hole size may not allow sufficient contact with the insecticide (Del Río et al., 2014). Paradoxically, a potential benefit of the lack of a repellent effect of alphacypermethrin against Culicoides midges is that it could increase (or at least not decrease) the numbers of midges coming into direct contact with treated mesh, and thereby promote insecticidal efficacy.

Exposure of $C$. imicola to the alphacypermethrin-treated HDPE mesh in the contact bioassay resulted in a rapid insecticidal effect. The initial magnitude and rate of inducing mortality was greater after 3 min exposure compared to $1 \mathrm{~min}$ exposure of midges to the mesh. Subsequently, at $30 \mathrm{~min}$ post exposure both groups had reached maximal effect which was maintained up to the final assessment at $24 \mathrm{~h}$. A similar contact assay and exposure period to that reported by Papadopoulas et al. (2010) was used. The aim of the present study was, however, to assess the potential of alphacypermethrin-treated meshes for protecting containerised transport systems of horses, and not to screen the potential for direct treatment of horses with alphacypermethrin. Therefore it was elected to use a treated mesh, instead of hair clippings, for exposure of midges. Although the contact bioassay was done with nulliparous females, the results would not be expected to be different for parous females or males, however this was not investigated. As male midges are not generally abundant in light trap catches near hosts (Venter et al., 2009) the light trap results could vary for males.

Although mortality in the bioassay treated groups was consistently significantly higher than that of the control group, a study limitation was the relatively high mortality (up to 57\% after $24 \mathrm{~h}$ ) recorded in the control group. This was likely related to the use of field-collected midges and experimental handling procedures. Dehydration during the holding period and the effect of chilling (Nunamaker et al., 1996) as an immobilising method may have contributed to the high mortality in the control group. Improved holding conditions and alternate immobilisation methods such as $\mathrm{CO}_{2}$ (Meyer and 
Schmidtmann, 1979) need to be evaluated for use in future insecticide bioassays against Culicoides midges.

The alphacypermethrin formulation used is recommended by the World Health Organization Pesticide Evaluation Scheme for treatment of mosquito nets as well as indoor residual spraying (Banek et al., 2010) and efficacy and safety has been demonstrated for malaria control (Ansari and Razdan, 2002; 2003). However, welfare concerns associated with reduction of airflow and animal confinement, as well as practicality restricts the use of completely enclosed structures for housing livestock species and combinations of protective measures against Culicoides midges may therefore be required (Calvete et al., 2010). On the other hand, in horses (which are generally more accustomed to stabling) the use of complete barriers may be more feasible, providing that ventilation is not compromised. For example, a South African field study found that closing stables by meshing with gauze cloth resulted in a 14-fold reduction in the numbers of $C$. imicola and Culicoides (Avaritia) bolitinos Meiswinkel entering the stables (Meiswinkel et al., 2000).

In conclusion, the worldwide increase in the transportation of animals and products in freight containers may increase the risk of viruses being transported (Reiter, 2010). Whilst the available data on Culicoides midge involvement in vector-borne pathogen importation via transport networks is limited (Carpenter et al., 2009; Napp et al., 2013), standardised measures such as those recommended by the OIE for AHSV (World Organisation for Animal Health, 2013) should be in place to ensure the safe transportation of animals, especially if these animals are being moved through known infected areas or areas with unknown risk. Based on the positive results of the present study, HDPE mesh has potential to reduce exposure of housed horses to Culicoides midges, specifically $C$. imicola, during high risk periods for AHSV transmission, or during containerised transport. Additionally, treating this mesh with alphacypermethrin may increase the overall field efficacy thereof in reducing Culicoides midge biting rates. Meanwhile, further investigation of the specific mesh in reducing midge biting rate under field conditions and the effect of the mesh on ventilation of housing is required. 


\section{Acknowledgments}

We thank Dr Elisabeth Scheffer for assistance with sorting of insect catches. The Faculty of Veterinary Science research fund, Department of Companion Animal Clinical Studies, Equine Research Centre and the Mary Slack and Daughters Foundation are acknowledged for study funding.

\section{Conflict of interest statement}

The authors declare no conflict of interest.

\section{References}

Abbott, W.S., 1925. A method of computing the effectiveness of an insecticide. J. Econ. Entomol. 18, 265-267.

Ansari, M.A., Razdan, R.K., 2002. Operational feasibility and bio-efficacy of alphacypermethrin (Fendona) treated jute curtains to control urban malaria in a slum settlement of Delhi, India. Indian J. Malariol. $39,66-75$.

Ansari, M.A., Razdan, R.K., 2003. Bio-efficacy and operational feasibility of alphacypermethrin (Fendona) impregnated mosquito nets to control rural malaria in northern India. J. Vector Dis. 40, 33-42.

Backer, J.A., Nodelijk, G., 2011. Transmission and control of African horse sickness in The Netherlands: a model analysis. PLoS ONE 6, e23066.

Banek, K., Kilian, A., Allan, R., 2010. Evaluation of Interceptor long-lasting insecticidal nets in eight communities in Liberia. Malaria J. 9, 84.

Barnard, B.J.H., 1997. Some factors governing the entry of Culicoides spp. (Diptera: Ceratopogonidae) into stables. Onderstepoort J. Vet. Res. 64, 227-233.

Braverman, Y., Chizov-Ginzburg, A., 1998. Duration of repellency of various synthetic and plant-derived preparations for Culicoides imicola, the vector of African horse sickness virus. Arch. Vir. S. 14, 165174.

Calvete, C., Estrada, R., Miranda, M.A., Del, R.R., Borras, D., Beldron, F.J., Martinez, A., Calvo, A.J., Lucientes, J., 2010. Protection of livestock against bluetongue virus vector Culicoides imicola using insecticide-treated netting in open areas. Med. Vet. Entomol. 24, 169-175.

Carpenter, S., Mellor, P.S., Torr, S.J., 2008. Control techniques for Culicoides biting midges and their application in the U.K. and northwestern Palaearctic. Med. Vet. Entomol. 22, 175-187. 
Carpenter, S., Wilson, A., Mellor, P.S., 2009. Culicoides and the emergence of bluetongue virus in northern Europe. Trends Microbiol. 17, 172-178.

de Vos, C.J., Hoek, C.A., Nodelijk, G., 2012. Risk of introducing African horse sickness virus into the Netherlands by international equine movements. Prev. Vet. Med. 106, 108-122.

Del Río, R., Barcelo, C., Lucientes, J., Miranda, M.A., 2014. Detrimental effect of cypermethrin treated nets on Culicoides populations (Diptera; Ceratopogonidae) and non-targeted fauna in livestock farms. Vet. Parasitol. 199, 230-234.

Dyce, A.L., 1969. The recognition of nulliparous and parous Culicoides (Diptera: Ceratopogonidae) without dissection. J. Aust. Entomol. Soc. 8, 11-15.

Hoffmann, B., Scheuch, M., Hoper, D., Jungblut, R., Holsteg, M., Schirrmeier, H., Eschbaumer, M., Goller, K.V., Wernike, K., Fischer, M., Breithaupt, A., Mettenleiter, T.C., Beer, M., 2012. Novel Orthobunyavirus in Cattle, Europe, 2011. Emerg. Infect. Dis. 18, 469-472.

Lengeler, C., 2004. Insecticide-treated bed nets and curtains for preventing malaria. Cochrane DB. Syst. Rev. 2, CD000363.

MacLachlan, N.J., Guthrie, A.J., 2010. Re-emergence of bluetongue, African horse sickness, and other orbivirus diseases. Vet. Res. 41, 35.

MacLachlan, N.J., Mayo, C.E., 2013. Potential strategies for control of bluetongue, a globally emerging, Culicoides-transmitted viral disease of ruminant livestock and wildlife. Antiviral Res. 99, 79-90.

Meiswinkel, R., Baylis, M., Labuschagne, K., 2000. Stabling and the protection of horses from Culicoides bolitinos (Diptera: Ceratopogonidae), a recently identified vector of African horse sickness. Bull. Entomol. Res. 90, 509-515.

Meiswinkel, R., Venter, G.J., Nevill, E.M., 2004. Vectors: Culicoides spp. In: Coetzer, J.A.W., Tustin, R.C. (Eds.), Infectious Diseases of Livestock. Oxford University Press, Cape Town, pp. 93-136.

Mellor, P.S., 1992. Culicoides as potential orbivirus vectors in Europe. In: Walton, T.E., Osburn, B.I. (Eds.), Bluetongue, African Horse Sickness, and Related Orbiviruses. CRC Press, Boca Raton, Florida, pp. $278-283$.

Mellor, P.S., Boorman, J., Baylis, M., 2000. Culicoides biting midges: their role as arbovirus vectors. Annu. Rev. Entomol. 45, 307-340.

Meyer, R.E., Schmidtmann, E.T., 1979. A system for the induction and maintenance of anesthesia for Culicoides. Mosq. News 39, 797-799. 
Napp, S., Garcia-Bocanegra, I., Pages, N., Allepuz, A., Alba, A., Casal, J., 2013. Assessment of the risk of a bluetongue outbreak in Europe caused by Culicoides midges introduced through intracontinental transport and trade networks. Med. Vet. Entomol. 27, 19-28.

Nevill, E.M., Venter, G.J., Edwardes, M., 1992. Potential Culicoides vectors of livestock orbivirus. In: Walton, T.E., Osburn, B.I. (Eds.), Bluetongue, African Horse Sickness, and Related Orbiviruses. CRC Press, Boca Raton, Florida, pp. 306-313.

Nunamaker, R.A., Dean, V.C., Murphy, K.E., Lockwood, J.A., 1996. Stress proteins elicited by cold shock in the biting midge, Culicoides variipennis sonorensis Wirth and Jones. Comp. Biochem, Physiol. 113B, 73-77.

Page, P.C., Labuschagne, K., Nurton, J.P., Venter, G.J., Guthrie, A.J., 2009. Duration of repellency of N,Ndiethyl-3-methylbenzamide, citronella oil and cypermethrin against Culicoides species when applied to polyester mesh. Vet. Parasitol. 163, 105-109.

Papadopoulos, E., Rowlinson, M., Bartram, D., Carpenter, S., Mellor, P., Wall, R., 2010. Treatment of horses with cypermethrin against the biting flies Culicoides nubeculosus, Aedes aegypti and Culex quinquefasciatus. Vet. Parasitol. 169, 165-171.

Paweska, J.T., Venter, G.J., 2004. Vector competence of Culicoides species and the seroprevalence of homologous neutralizing antibody in horses for six serotypes of equine encephalosis virus (EEV) in South Africa. Med. Vet. Entomol. 18, 398-407.

Reiter, P., 2010. The standardised freight container: vector of vectors and vector-borne diseases. Rev. Sci. Tech. $29,57-64$.

Scheffer, E.G., Venter, G.J., Labuschagne, K., Page, P.C., Mullens, B.A., MacLachlan, N.J., Osterrieder, N., Guthrie, A.J., 2012. Comparison of two trapping methods for Culicoides biting midges and determination of African horse sickness virus prevalence in midge populations at Onderstepoort, South Africa. Vet. Parasitol. 185, 265-273.

Snedecor, G.W., Cochran, W.G., 1980. Statistical methods. Iowa State University Press.

Tarlinton, R., Daly, J., Dunham, S., Kydd, J., 2012. The challenge of Schmallenberg virus emergence in Europe. Vet. J. 194, 10-18.

Venter, G.J., Labuschagne, K., Hermanides, K.G., Boikanyo, S.N.B., Majatladi, D.M., Morey, L., 2009. Comparison of the efficiency of five suction light traps under field conditions in South Africa for the collection of Culicoides species. Vet. Parasitol. 166, 299-307. 
Venter, G.J., Labuschagne, K., Boikanyo, S.N., Morey, L., Snyman, M.G., 2011. The repellent effect of organic fatty acids on Culicoides midges as determined with suction light traps in South Africa. Vet. Parasitol. $181,365-369$.

Venter, G.J., Majatladi, D.M., Labuschagne, K., Boikanyo, S.N., Morey, L., 2012. The attraction range of the Onderstepoort 220V light trap for Culicoides biting midges as determined under South African field conditions. Vet. Parasitol. 190, 222-229.

Venter, G.J., Paweska, J.T., Van Dijk, A.A., Mellor, P.S., Tabachnick, W.J., 1998. Vector competence of Culicoides bolitinos and C. imicola (Diptera: Ceratopogonidae) for South African bluetongue virus serotypes 1, 3 and 4. Med. Vet. Entomol. 12, 101-108.

Viennet, E., Garros, C., Rakotoarivony, I., Allène, X., Gardès, L.t., Lhoir, J., Fuentes, I., Venail, R., Crochet, D., Lancelot, R., Riou, M., Moulia, C., Baldet, T., Balenghien, T., 2012. Host-Seeking Activity of Bluetongue Virus Vectors: Endo/Exophagy and Circadian Rhythm of Culicoides in Western Europe. PLoS ONE 7, e48120.

World Organisation for Animal Health, 2013. Infection with African horse sickness virus. In: Terrestrial Animal Health Code, OIE, Paris. Available at http://www.oie.int/fileadmin/Home/eng/Health standards/tahc/2010/chapitre 1.12.1.pdf. (accessed on 26 November 2013). 